\title{
El ciclo de las políticas públicas interculturales: paradojas $\underline{\text { político-antropológicas }^{1}}$
}

The intercultural public policies cycle: political-anthropological paradoxes

\author{
Alfonso Barquin Cendejas ${ }^{2}$ \\ Instituto Nacional de Antropología e Historia, México D. F., México \\ https://orcid.org/0000-0003-1729-9806
}

Artículo de reflexión

Fecha de recepción: 30 de marzo de 2019

Fecha de aceptación: 14 de mayo de 2019

\section{Para citar este artículo}

Barquín Cendejas, A. (2019). El ciclo de las políticas públicas interculturales: paradojas político-antropológicas. Campos en Ciencias Sociales, 7(2), 147-xx. DOI: https://doi.org/10.15332/25006681.5105

1. Este artículo de reflexión es parte de los resultados teóricos de la línea de investigación Poder y políticas públicas: la instrumentación de los marcos legales del Estado, que se desarrolla en la Dirección de Etnología y Antropología Social del INAH (México), como una búsqueda por caracterizar y analizar los efectos del ejercicio del poder político del Estado en su dimensión cultural, dentro del marco de la antropología política.

2. Doctor en Ciencias Antropológicas de la Universidad Autónoma Metropolitana de México. Investigador titular en la Dirección de Etnología y Antropología Social del Instituto Nacional de Antropología e Historia de México. Correo electrónico: albarcen@gmail.com 


\title{
RESUMEN
}

En el presente trabajo se realizó una reflexión sobre las paradojas que aparecen en la implementación de políticas públicas de carácter intercultural. Para la antropología como disciplina aplicada, resulta fundamental considerar los efectos que surgen en la implementación de políticas que no convergen con lo planeado y generan efectos distintos o contrarios al esperado. Con el apoyo de herramientas teóricas de otras disciplinas sociales y sin perder el hilo de lo cultural, se expuso un panorama para definir y tipificar efectos paradójicos ilustrados con ejemplos de políticas con componente intercultural, esto es, aquellas en las que la diferencia cultural aparece como un antagonismo político.

Palabras clave: antagonismo, interculturalidad, paradoja, políticas públicas.

\begin{abstract}
The present work makes a reflection on the paradoxes that appear in the implementation of intercultural public policies. For anthropology as an applied discipline, it is fundamental to consider the effects that arise in the implementation of policies, which are not convergent with the planned but different or contrary to the expected one. With the support of theoretical tools from other social disciplines and with the cultural dimension as a guiding thread, a panorama is presented to define and typify paradoxical effects illustrated with typical examples of intercultural policies, that is, those in which the cultural difference appears as a political antagonism.
\end{abstract}

Keywords: Paradox, interculturality, public policies, antagonism. 


\section{INTRODUCCIÓN}

La política como eje de la organización humana se nos presenta como un sistema de gestión de los antagonismos sociales sobre temas comunes. Es decir, para una parcela de la realidad sobre la que confluye un grupo social, existen visiones distintas para aproximarse a su manejo. Tenemos así esperanzas comunes con expectativas divergentes. Esta paradoja constitutiva de lo político retoma las visiones antagónicas de Hannah Arendt (1997) y de Carl Schmitt (1998), pues la primera ve la esencia de la política como composición, mientras el segundo destaca la oposición como rasgo distintivo. La contradictoria circunstancia a la que se enfrentan los individuos, puede en múltiples casos solucionarse total o parcialmente mediante un proceso político con el cual se fijan mínimos aceptables, se cede en algunos temas, se imponen otros y se logra cierto grado de estabilidad hasta que alguna de las partes manifieste su inconformidad con el arreglo alcanzado. Si fuese imposible el arreglo la ruptura aparece como un hecho normal de la organización social.

La coexistencia de grupos humanos con culturas diferentes se une de manera sorprendente con la definición de política presentada. Frente a un tema que resulta compartido por dos culturas, florecen no solo visiones divergentes sino que en ciertos aspectos las posiciones son ininteligibles pues frecuentemente, las razones resultan incomprensibles a la luz de las estructuras culturales del otro. Hay que señalar que para una cultura los antagonismos internos son claramente lógicos: comparten el tema y los insumos para su aproximación, pero se confrontan en la distribución o los efectos de cada solución. En los antagonismos entre culturas, las divergencias nacen de la estructura misma de aproximación y de la importancia asignada a ese tema o a otros no considerados; cada grupo se acerca desde un punto de vista distinto al del otro o desde una ponderación diferente. Pueden no compartirse relevancia del tema, insumos, distribuciones o efectos. No son, sin embargo, más o menos políticos los antagonismos entre culturas. Los resultados del proceso político entre culturas pueden terminar como se mencionó: en un consenso pleno, en un acuerdo negociado, en distintos grados de imposición o en la ruptura parcial o total entre las partes.

La cuestión de las paradojas que surgen en la implementación de políticas interculturales mana de las características expuestas arriba. En esta reflexión se 
presentan algunos resultados paradójicos que nacen cuando un Estado genera políticas públicas que se aplican en una comunidad de matriz cultural distinta a la de su población mayoritaria. El objetivo no es mostrar que los caminos antagónicos de la política culminan en ruptura o parálisis, en otras palabras, que como son malos resultados "no hay que hacer nada y dejar las cosas como están". Se trata de exponer que en la elaboración de políticas con diversidad cultural poco se consideran tanto las tensiones contradictorias de un diseño de implementación, como la posibilidad de resultados divergentes o contrarios al espíritu de lo planeado, es decir, paradójicos.

En primer lugar hay que aclarar lo que significa en esta exposición la idea de paradoja. Desde luego que en su sentido más llano la paradoja es una contradicción lógica, pero aquí se enfoca hacia un aspecto central de la vida social: los antagonismos constitutivos. Se puede argumentar que existen tantas opiniones como individuos, sin embargo, en comunidad se generan reducciones a los aspectos más característicos en la búsqueda por mantener la unidad del grupo: la paradoja reside en la búsqueda incesante de las pulsiones individuales y simultáneamente, en la búsqueda de la contención de tales pulsiones para lograr la unidad colectiva. Es evidente que desde el punto de vista de los involucrados, se puede imaginar la posibilidad de llegar a una solución de las contradicciones para consolidar de manera plena la idea de comunidad. Sin embargo, la idea de una comunidad plena y sin antagonismos que elimine las contradicciones de una vez y para siempre, es llanamente una utopía. Ernesto Laclau y Chantal Mouffe (2004) lo han expuesto de manera por demás acabada: la relación entre particularidades sociales que buscan imponer su punto de vista a toda la comunidad es una expansión de carácter hegemónico pero ineficiente, pues nunca una particularidad puede transformarse plenamente en universal; siempre es inestable y reversible por el carácter inerradicable de los antagonismos ( $\mathrm{p}$. 17). Más aun, en una obra posterior, Mouffe expone el dilema en la dinámica de las democracias liberales contemporáneas al mostrar la paradoja de tal régimen político, pues el contenido liberal -imperio de la ley, defensa de los derechos humanos y respeto a la libertad individual - se confronta con las ideas nucleares de la democracia (igualdad social, identidad entre gobernantes y gobernados y soberanía popular). Es una lucha que desde su punto de vista no solo no acaba, sino que no debe acabar y que constituye un régimen que denomina como "agonista". Para ella, el pluralismo agonista, “... no consiste en eliminar las pasiones de la esfera de lo público para 
hacer posible el consenso racional, sino en movilizar esas pasiones en la dirección de los objetivos democráticos" (Mouffe, 2012, p. 116). Así, lo que llama "la paradoja democrática" se asienta en la idea de que es legítimo establecer límites a la soberanía popular para garantizar la libertad individual.

Asimismo, las paradojas resultan del desarrollo de una acción que, esperando un resultado óptimo de los procedimientos efectuados, culmina en efectos divergentes o contradictorios a lo planeado. Por lo tanto, en la implementación de políticas interculturales, las paradojas rondan el proceso, pues sumado al frecuente fallo en los efectos finales de una política, se presenta la posibilidad de contradicciones insalvables ya que desde dos posiciones culturales diferentes, las evaluaciones pueden ser antagónicas.

¿Por qué resulta de interés antropológico un punto de vista que mana de la filosofía política para el análisis de las políticas públicas de naturaleza intercultural? Básicamente porque expone de una forma sencilla el hecho de que en política siempre existen tensiones, siempre deseos de expansión hegemónica, siempre insatisfacciones con los resultados conseguidos y siempre deseos de cambio o profundización del estatus. En los dilemas de las políticas públicas interculturales, a las tensiones normales de lo político se suma una adicional: la incompatibilidad de las estructuras culturales.

Juzgar las formas, contenidos, métodos de aplicación y evaluación de resultados de las políticas que aplica el Estado en una comunidad de matriz cultural diferente, revela tensiones antagónicas y paradojas diversas que vale la pena señalar. En primer lugar está la idea de la naturaleza exógena de las políticas que aplica el Estado. Como la máxima organización política en un territorio -según la idea de Max Weberlas políticas originadas en los mandatos constitucionales tienen, por definición, que ser aplicadas de manera general en el territorio mentado. Sin embargo, para una sociedad nacional en la que coexisten divergencias culturales, resulta complejo aplicar a plenitud lo que de manera general sería el credo de la interculturalidad: comprender la especificidad de cada cultura, respetar sus razones o características particulares y animar la conservación de dichas tradiciones culturales, todo ello en un contexto de convivencia entre dos o más culturas en el que ninguna de ellas 
imperaría. Ideas en este sentido se pueden encontrar en Bartolomé (2006), Bertely (2009), CGEIB (2006 y 2017), Dietz (2003), Matto (2008) y Díaz Polanco (2006).

Esta forma descrita, que supone un Estado en el que la base territorial que le da origen carece de los efectos de la homogeneidad poblacional, apunta a un origen a-cultural del Estado. En otras palabras, el nacimiento de los Estados reales como organizaciones políticas está determinado por cierto núcleo homogéneo de población que le da forma y lo ordena. Es bien cierto que la idea del monopolio de la fuerza sobre el territorio que lo hospeda, empuja al Estado a englobar las formas que no empalman con su matriz de origen, en este caso el de la cultura que lo genera. No obstante, la humanidad en su esfuerzo por la convivencia va ajustando las divergencias culturales que enfrentan a los distintos grupos, generando patrones normativos para contener los excesos en el manejo de dichas heterogeneidades. Las definiciones constitucionales sobre la convivencia entre culturas -como ocurre en general en el caso latinoamericano y en particular en el caso mexicano-impulsan un avance hacia los temas del respeto, la comprensión y el mantenimiento de la diversidad cultural. Sin embargo, es un Estado el que empuja esa revisión "intercultural” y de ahí surge la primera paradoja: el Estado impone un orden político sobre toda la población en general; ese es su carácter. No distingue entre individuos de una cultura A o $\mathrm{B}$ para la definición de sus lineamientos generales, ni renuncia a su atribución de ejercer el poder político que le da sentido. Su matriz cultural está definida por una de esas fracciones y partiendo de esta, ha trabajado para aminorar o contener esa presión homogeneizadora. De esta precisa manera es que se desarrolló la reforma del artículo segundo de la Constitución Política de los Estados Unidos Mexicanos (2019), obligando al Estado mexicano a reconocer su dominación hegemónica y por lo tanto, eliminarla en la búsqueda de una convivencia no hegemónica y de carácter intercultural.

La peculiar circunstancia es que muchos de los temas operativos del Estado y de su poder político están más allá de las formas culturales particulares: el federalismo fiscal, el electoral, la regularidad energética o de comunicaciones, los avances tecnológicos, la unidad monetaria, las certificaciones académicas o laborales, la redistribución económica, etcétera. Son fuerzas que impactan el interior de las comunidades con cultura diferente y que difícilmente se les podría dar otra forma, salvo que se 
renunciara a insertarse en el efecto. Así, la paradoja surge por la búsqueda de una igualdad absoluta en la atención estatal en un contexto de especificidad cultural regional, donde la demanda de autonomía impacta antagónicamente las definiciones de lo que es el Estado en su sentido más puro y más allá del accidente cultural. De hecho, la solución política que la humanidad ha dado a este problema es justo la constitución de Estados-nacionales plurales y distintos del monocultural original, buscando resolver el tema de la atención acorde con las necesidades internas de una comunidad; Cataluña, Sudán o los Balcanes son una muestra reciente de esos problemas y soluciones. Como dice Claude Levi-Strauss:

Sin duda nos acunamos con el sueńo de que la igualdad y la fraternidad reinarán un día entre los hombres, sin que se comprometa su diversidad. Pero si la humanidad no se resigna a transformarse en la consumidora estéril de los únicos valores que supo crear en el pasado, capaz solamente de dar a luz obras bastardas, invenciones groseras y pueriles, deberá aceptar que toda creación verdadera implica una cierta sordera a la llamada de otros valores, pudiendo llegar hasta el rechazo y aún a su negación. Porque no se puede, a la vez, fundirse en el goce del otro, identificarse con él y mantenerse diferente. Plenamente lograda, la comunicación integral con el otro, condena en un plazo más o menos breve la originalidad de su creación y de la mía. (p. 141).

Es decir, la paradoja surge de un deseo de mantener lo externo fuera de lo interno (el Estado fuera de la comunidad) y, sin embargo, generar políticas externas que satisfagan las necesidades internas, sin atender las reglas que el Estado generó para sí o, más dramático aún, que las disposiciones de lo interno modifiquen al órgano externo para su funcionamiento total. Esta visión, cuyo género más problemático es el ejercicio de poder del Estado vía las políticas, tiende a ignorar las complejidades del proceso político que, como aseveran Laclau y Mouffe, emergen de la búsqueda por la hegemonía de una visión particular sobre el universo político total. Asimismo, que desconociendo la circunstancia de los antagonismos inerradicables, se postula un proyecto en el que la intersección entre culturas esté vacía de poder, paradoja máxima, pues si el poder es un elemento constitutivo de la conformación de una cultura, resulta insostenible establecer un espacio social a-politico, libre de este elemento central de la socialización. Más bien habría que caracterizar a la interculturalidad en 
términos de poder, como un espacio agonístico donde hay una lucha continua y no destructiva entre dos o más culturas, en vez de imaginar un universo sin antagonismos culturales o ejercicio de poder, lo que conforma una visión completamente utópica, pues al no existir los antagonismos, lo político como tal llegaría a su fin.

Para presentar los temas sobre los accidentes paradójicos en el desarrollo de las políticas públicas, en este apartado se desarrollan algunas de las complejidades que se plantean desde la teoría de las políticas públicas reseñando los puntos problemáticos y cómo existen posiciones antagónicas sobre una misma idea.

Lo primero sería destacar que una política pública es: "una intervención deliberada del Estado para corregir o modificar una situación social o económica que ha sido reconocida como problema público. También suele llamarse con ese nombre a las decisiones transversales que regulan la actuación interna de los Gobiernos" (Merino, 2013, p. 17). Aparecen tres ideas que conviene llevar en mente y que ya se habían delineado anteriormente: la actuación del Estado para modificar una situación social; su capacidad para lograrlo vía el ejercicio del poder político, y la decisión de elegir un curso específico frente a varios posibles. Para conseguir lo anterior, conviene bosquejar que existe todo un cuerpo teórico y de diseńo sobre las intervenciones, la ejecución y la evaluación.

El primer tema se refiere al marco teórico propio de las fases o ciclos de las políticas públicas. En general se tienen aceptados cuatro grandes momentos: 1) El metaanálisis referido a la idea sobre lo político y su marco normativo; 2) el mesoanálisis, concerniente a la definición del problema y de la agenda; 3) el análisis y la toma de las decisiones y, 4) el momento de la implementación y la evaluación de resultados (Parsons, 2007).

Con relación al primer punto solo referiremos que toca aspectos sobre la naturaleza de lo político ya tratados en el inicio de esta reflexión y que, si bien no agotaron el tema, mostraron algunos de los aspectos más útiles para el argumento. 
Respecto al segundo punto, tampoco se abundará mucho pues atañe al mecanismo por el cual, en este caso, las relaciones entre culturas en el México contemporáneo se definieron como problema y se colocaron en la agenda de manera prioritaria luego de la reforma del año 2001 al artículo segundo de la Constitución mexicana. Dado el vasto cuerpo bibliográfico sobre el particular no es necesario abundar sobre el asunto. Para lo que estamos analizando, sí es necesario referirse al tercero y cuarto aspectos, pues exponen las dificultades en el diseńo y la toma de decisiones interculturales.

Uno de los aspectos en los que se gasta la mayor cantidad de tinta con relación a la racionalidad para decidir un curso de acción y aplicarlo, se refiere a qué tanta confianza se puede tener en las predicciones lógicas de conducir la acción social vía una política pública. El tema de mayor controversia que surge es sobre si es posible el diseño e implementación racional de un proceso de políticas. Aquí hay un gran debate sobre los límites de cuánto y cómo se puede prever. Proviniendo de la tradición racionalista de la Modernidad, y teniendo como eje la planeación en tiempos de la Segunda Guerra Mundial, en Estados Unidos se gestó una gran confianza sobre la planeación racional de políticas que preveía todos los imponderables posibles (Orozco, 1978; Collins, 1996). En 1959 se publicó un artículo que cuestionó dicha posibilidad colgado del proyecto de racionalidad limitada de Herbert Simon. La ciencia de salir del paso de Charles Lindblom ([1959] 2000) expuso una serie de cuestionamientos sobre los límites racionales para tener el control total de los efectos de una política pública. Inaugurando un programa conocido como "incrementalismo", expresó serias dudas sobre el control de las políticas y las limitaciones de tiempo para la obtención de información y su análisis, las limitaciones de recursos y las fallas de los modelos teóricos para determinar el proceso de causa-efecto. Por ello argumentó que los efectos de las políticas deberían ser limitados e ir revisando cada pequeño paso para ver si el efecto era el deseado y, en caso contrario, corregir hacia el futuro. La crítica no tardó en llegar, fundamentalmente en cuanto a que si una sociedad era desarrollada como la estadounidense, los pequeños pasos pueden ser un buen programa. Sin embargo, en países con situaciones de profundo atraso, los pequeños pasos pueden no atacar el problema de raíz, aun cuando una gran intervención corra el riesgo de fallar (Dror, 2000). Posteriormente, se generó una visión que postulaba un diseño y ejecución "combinada", es decir, planeando "en grande" y ejecutando "en pequeño" para aproximarse con más precaución al resultado deseado (Etzioni, 2000). 
Aquí es que de nuevo aparecen efectos paradójicos. Por un lado es cierto que en la sociedad, el cálculo de efectos de una cadena causal extensa es imposible, así que parece más razonable intentar los pequeños efectos, aunque por otro lado resultan insuficientes y sospechosos para arreglar los "grandes problemas nacionales". De tal suerte que una aproximación combinada parece una solución que, sin embargo, corre el peligro de profundizar las contradicciones de ambos esquemas en una solución intermedia que pudiera ser el peor de los escenarios al incurrir en los defectos de ambos modelos. Una advertencia muy saludable es la que plantea Giovanni Sartori (2002) a propósito de estas dos visiones. Precisa que la pequeña intervención al margen de las ventajas reseńadas, presenta efectos que pueden no ser reales por la dinámica controlada o por el plazo de aplicación y que al ser extendidos, o pierden ímpetu efectivo o el resultado irá mutando hacia lo imprevisto o lo perverso. Para el caso de la gran intervención, las expectativas son muy ambiciosas y se alinean con la forma "natural" de imaginar una política. El problema es que el paso de una escala a otra no es mecánico, los medios se tornan altamente costosos, las dinámicas son de largo plazo y los efectos no son visibles de manera inmediata, por lo cual o se extinguen los medios o no funciona como se previó, y cuando se advierte la falla los efectos ya no son corregibles en atención a la magnitud de la intervención.

Un suceso particular de este último caso que expone Sartori, es el interesante fenómeno en el que los medios rebasan a los fines, es decir, que en la búsqueda de un efecto ambicioso la aplicación tan grande de ciertos medios puede irse transformando en un fin en sí mismo por el esfuerzo social que se requiere para su ejecución. Así, si el efecto no ocurre y se procuran cada vez más medios, en un punto de difícil diagnostico o retorno, estos "rebasan" a los fines produciendo efectos no deseados o perversos con el objetivo inicial. Caso parecido al del efecto que unos fines tienen sobre otros, no solo por la "distracción" de medios, sino porque muchos temas en sociedad suponen pares antagónicos de acción.

Se mencionó al inicio que liberalismo y democracia son un binomio paradójico, pues la ampliación en una dirección supone la reducción en otra. Sin abundar, solo se destaca que en el tema de la interculturalidad, el hecho de que a más autonomía de decisión en la comunidad cultural diferente y minoritaria, más grande será la separación respecto de la sociedad mayor a la que se quieren integrar plenamente. 
Para concluir esta sección se presentan de manera muy sintética las ideas más importantes que Robert Merton (1936) desarrolló en su artículo sobre las consecuencias no intencionales que surgen en la acción intencional. Trabajo que conserva su validez y frescura más de 80 ańos después de ser publicado y que por primera vez en la sociología abordó el tema de manera expresa, aunque ya antes algunos como Maquiavelo, Adam Smith, Karl Marx o Max Weber se habían ocupado del tema. Sin tener influencia del planteamiento de la racionalidad limitada que se reseño arriba -el artículo de Merton apareció años antes-, su visión está fundada en un sano entendimiento de los efectos de las normas sociales y su dimensión efectiva en la operación de las instituciones humanas. Es decir, no es que lleve al máximo el programa racional detectando entonces las fallas ineludibles para después postular una moderación por desconfianza de lo racional. Más bien aborda lo que en la ejecución de programas colectivos de acción representan las trabas institucionales, las normativas y y por qué no decirlo, las culturales. La primera objeción se refiere al estado social del conocimiento de los fenómenos de causa-efecto. No se refiere a una falla en términos de racionalidad, sino a los distintos grados de conocimiento social sobre un fenómeno que, al ser diferentes o imperfectos, producen actuaciones divergentes supuestas como homogéneas. Esta diferencia lleva a que los comportamientos enlazados en una cadena de aplicación-ejecución como la que ocurre en una política pública produzcan resultados no previstos, inocuos o dañinos para la política instrumentada. Una segunda dimensión se refiere llanamente al error que ocurre en los cursos de acción, independientemente de la buena voluntad de los actores, que arroja resultados distintos a lo planeado y que sumados socialmente producen resultados no deseados. Los hábitos constituyen una de las fuentes más profundas de error pues ante el cambio de circunstancias, los procedimientos habituales se mantienen constantes arrojando resultados no considerados. Como tercera consideración, plantea Merton el imperio de los intereses inmediatos que generan, en el individuo o los grupos, renuencia para ejecutar un curso de acción en el que no alcanzan a ver los resultados o cuyos efectos iniciales parecen contrarios a sus intereses de corto plazo. En este caso la modificación hacia los resultados inmediatos es una actitud comprensible. La dimensión políticopartidista de las políticas está fuertemente distorsionada por esta variable. Un cuarto aspecto muy convergente con el tema de las políticas interculturales se refiere al argumento de Merton respecto de la ejecución de un curso de acción orientado por normas o valores, independientemente de sus consecuencias objetivas, atendiendo 
a lo que Weber (1995) llamaba la "ética de la convicción" en oposición a la "ética de la responsabilidad". Finalmente, un tema por demás interesante se refiere al efecto mismo que una política introduce en el ambiente y que de hecho modifica las expectativas sociales. Vale la pena citar su aseveración: "predicciones públicas del desarrollo de un futuro social, frecuentemente no son alcanzadas precisamente porque la predicción se ha convertido en un nuevo elemento en la situación concreta, tendiendo así a cambiar el curso inicial de desarrollo" (Merton, 1936, p. 903). Esto es lo que esta autor llama la "profecía suicida": cuando una sociedad reacciona de acuerdo a sus intereses o valores a la pronunciación de un curso político por venir para defender o aprovechar lo que considera valioso; esta respuesta que es previa o simultánea a la instrumentación de la política, distorsiona los efectos previstos pues genera un tipo de respuesta que ignoró en su diseño, la respuesta misma.

¿Qué conclusiones se pueden extraer de los argumentos anteriores? Una idea inmediata y evidentemente errónea es que no es factible lograr efectos sociales deseados mediante las políticas públicas. Nada más equivocado. El ejercicio del poder político tiene efectos y en múltiples ocasiones son efectos convergentes con los cursos de acción planeados. Más bien la lección aquí es que a pesar de que el poder sea ejercido de manera plena, sus resultados efectivos o bien no son del todo convergentes con lo planeado o su evaluación de largo plazo no muestra la eficacia deseada. Esto es debido a un efecto muy comprensible en el que si una política se impone y ejecuta en primera instancia, genera una ilusión de efectividad sobre las consecuencias finales. Dicha ilusión deriva de que la eficacia inmediata del ejercicio del poder, se asemejaría a un control total de la realidad social.

Otro resultado interesante es que existe una paradoja difícil de resolver entre la dimensión racional intensiva y de largo aliento y las consideraciones de tipo incremental y de corto plazo. La tensión sobre qué tanto de cada programa hay que incorporar es constante, pues son definiciones antagónicas y por lo tanto su balance no es del todo claro en el horizonte de planeación e implementación.

Por lo tanto, el objetivo de exponer las componentes paradójicas en la instrumentación de políticas públicas estatales es el de destacar, desde una dimensión teórica y formal, el frecuente exceso de confianza y las ilusiones de que los cursos de acción 
gubernamental van a atracar de manera segura en el puerto de las soluciones exitosas. En las discusiones sobre los efectos de las políticas públicas interculturales en las que al autor de esta reflexión le ha tocado participar, hay consistente proclividad a tener gran confianza en los supuestos político-normativos que soportan la ejecución de las políticas interculturales y resulta en cambio poco frecuente, la manifestación de una sana desconfianza sobre la cadena de causalidades que supone una determinada política. Como lo expondría Merton, se percibe una gran confianza en que el apego a ciertas normas que son satisfactorias de manera subjetiva, bastarán para que el resultado de las políticas culmine en los efectos imaginados.

En la siguiente y última sección, se van a enlazar los análisis efectuados con algunos supuestos orientadores de las políticas públicas interculturales con el ánimo de detonar una reflexión más cauta de hasta dónde las políticas implementadas están avanzando hacia el éxito deseado y cuándo es que se encaminan hacia efectos no considerados que pueden ser antagónicos al deseo que las motivó. Hay que señalar que en una revisión de la literatura sobre las políticas públicas y la interculturalidad, buscando su dimensión paradójica, se observa fundamentalmente una orientación hacia los aspectos normativos - lo que cimenta y debe incluir una política intercultural- o hacia los aspectos correctivos -lo que está mal implementado de cara a la dimensión normativa- de tal manera que no abordan los aspectos técnicos o políticos insolubles y por ende, paradójicos (Ávila, 2018; CESOP, 2015; Cruz, 2014 y 2015; Fleckman, Dal Corso, Ramírez, Begalieva y Johnson, 2015; Huerta, 2016; Mendoza, 2013; Ministerio de Cultura, 2009; Monroy, 2017; Rodríguez, 2015; Zapata-Barrero, 2015).

Habiendo ya comentado los aspectos problemáticos de la relación entre lo externo y lo interno en la acción del Estado, se buscará ilustrar de manera esquemática cómo se relacionan los anteriores con algunos ejemplos concretos, evidenciando siempre su carácter paradójico. Inicialmente se mencionará el hecho de que las políticas interculturales, como se ven al menos en México, suponen en su operación e implementación el mantenimiento de cierta asimetría entre el Estado y las comunidades con diferencia cultural. Como bien lo expone Guillermo Bonfil en un esquema muy conocido sobre la forma de tomar decisiones en una cultura -propias o impuestas- y los elementos culturales con los que se toman -propios o ajenos-, el 
argumento desemboca en lo deseable que resultaría que una cultura en particular se manejara de manera autónoma, es decir, tomando decisiones propias con elementos culturales propios (Bonfil, 1991, pp. 49-57). A la vista de la historia política de la humanidad, la solución que resulta de este planteamiento es la conformación de un Estado-nación, que justo condensa autonomía de decisión con regularidad cultural. De ahí que se resalte lo paradójico de intentar políticas estatales externas que respeten plenamente la vida cultural interna en una sola unidad política. Un complemento a este argumento es lo referido a un espacio de intersección entre dos culturas vacío de poder-, que se supone es lo intercultural. A la vista del argumento anterior, es imposible sacar el poder político de la ecuación salvo en el caso de que las dos entidades culturales sean cada una entidades autónomas como propone Bonfil, lo que cambiaría sus relaciones de interculturales a inter-nacionales.

Tal vez uno de los ejemplos más interesantes de las políticas de naturaleza intercultural, son las relativas a la salvaguardia del patrimonio cultural inmaterial (PCI), fundamentadas en la Convención para la Salvaguardia del Patrimonio Cultural Inmaterial (Unesco, 2004). Existe una larga y compleja trayectoria para la culminación de esta convención, cuyo objetivo es la generación de políticas para la salvaguardia del PCI (Rebollo, 2017). De estas políticas, una de las más interesantes se refiere a las paradojas originadas por las declaraciones de inclusión en las listas representativas del PCI que elabora la Unesco. Generadas en un acuerdo internacional para la preservación de las culturas tradicionales y en particular de las comunidades que quedaron enclaustradas en Estados hegemónicos con una cultura distinta, el objetivo de la convención es la elaboración de listas de elementos culturales que requieren ser preservados con la consecuente implementación de políticas públicas de salvaguardia. Sobre lo anterior, se mostrarán algunos efectos paradójicos detonados por la emisión de una declaratoria de patrimonio cultural inmaterial. El deseo de generar políticas de salvaguardia se emparejó con la convicción de aprovechar tales elementos para detonar el desarrollo sostenible de las comunidades deprimidas (Unesco, 2004, p. 2). Así las cosas, los Estados parte de la declaración y en particular las unidades políticas regionales, buscaron la emisión de declaratorias con el foco puesto en la dimensión del desarrollo económico generado, sobre todo en su componente turístico. Aunado al efecto de llamar la atención pública y gubernamental sobre el fenómeno y la posibilidad de canalizar fondos tanto estatales como privados, aparecieron algunos efectos perversos que vale la pena comentar. 
En primer lugar está el hecho de la competencia internacional entre países, por contar con un número importante de declaratorias. Emitido el documento, se ignoran los compromisos para la salvaguardia quedando como núcleo único el mero registro, lo que significa para los actores nacionales, regionales y algunos locales, una sensación de orgullo. El mayor esfuerzo respecto de la declaratoria se orienta a entrar en la lista representativa y no en la implementación del plan de salvaguardia, lo que banaliza el elemento que se quiere enaltecer. Como se mencionó arriba, es una respuesta de los intereses inmediatos sobre las necesidades de largo plazo.

En segundo lugar, apareció el fenómeno no planeado y perverso de la masificación turística por el conocimiento del elemento en salvaguardia. La curiosidad por conocer dicho patrimonio detonado por la publicidad privada y gubernamental, en algunos casos ha tomado dimensiones verdaderamente inmanejables, lo que amenaza con diluir la esencia del elemento por las presiones para adaptar la dinámica interna del fenómeno a las necesidades de la masa de personas que lo quieren "disfrutar". Como un ejemplo de lo anterior tenemos el caso emblemático que ocurre en la "danza de los parachicos" (Bolaños y Barquín, 2014), fiesta tradicional originada en el siglo XVII en la que se agradece una curación milagrosa con música y bailes, que se lleva a cabo a principios de enero en la ciudad de Chiapa de Corzo en el estado de Chiapas (México), y que presenta dimensiones inmanejables de turistas que afectan el desarrollo de los festejos en su vertiente tradicional, al grado de que el "patrón de los parachicos" está impedido para comunicarse con sus subordinados respecto a un mejor desarrollo de la fiesta. Otro caso es el de la celebración del "Día de Muertos" (Pérez Ruiz, 2014), festividad en la que se recibe a las almas de los parientes difuntos que regresan a visitar a los vivos; se desarrolla en la ciudad de Pátzcuaro, Michoacán, el 1 y 2 de noviembre de cada ańo, con la creciente presión por el turismo y la mercantilización que rompe con la necesaria intimidad que la celebración requiere. Las consecuencias expuestas en estos ejemplos exponen dos de las componentes teóricas señaladas arriba. Por un lado, considera la advertencia de Sartori sobre la complejidad de parar un efecto macroscópico cuando se detectan consecuencias no deseadas, y por otro, toma el efecto de la profecía suicida que mencionó Merton, pues la declaración pública de la intención de preservar un elemento cultural en riesgo, lo pone de manera inmediata ante una mayor amenaza por el deseo masivo de publicitar y de conocer una tradición que se está perdiendo. 
Un último efecto por señalar es el de la unidad de la comunidad portadora de dicho patrimonio. Se entiende que el patrimonio es generado por una comunidad que preserva una práctica cultural más o menos homogénea. Esta regularidad cultural y la unidad interna que supone, deriva en que cuando el Estado pretende registrar una candidatura para elaborar un plan de salvaguardia, se debe consultar a los portadores sobre si están de acuerdo con los términos a implementar por la obligatoriedad constitucional de diseñar y operar las políticas conjuntamente con ellos, como reseńa el apartado B del artículo segundo de la Constitución mexicana. Sin embargo, esta regularidad cultural no considera la heterogeneidad económica, política, generacional o religiosa, por citar algunas, y que desgajan la unidad interna de la comunidad, sobre todo porque estos índices tienen una conexión profunda con la sociedad externa; es decir, se parecen más a lo externo que a lo interno. Estas divergencias pueden derivar en que una parte de la comunidad apruebe el registro del patrimonio cultural y las políticas de salvaguardia y otra no. El resultado efectivo es de nueva cuenta paradójico, pues si el Estado resuena con la fracción que aprueba la declaratoria es factible considerar que llevará su plan a cabo, pero generando un rechazo mayúsculo en la otra parte, lo que en términos de la preservación del patrimonio es fatal, pues se da al traste con la expuesta homogeneidad cultural. Este es el caso de la pirekua, género musical de gran calidad y sensibilidad del pueblo purépecha que habita en el estado de Michoacán y que fue declarada como patrimonio cultural con la aprobación de solo una parte de la comunidad, desembocando en la inoperatividad de la política y la ruptura de la homogeneidad comunitaria (Flores Mercado, 2014).

Es decir, existe una situación conflictiva para el desarrollo de políticas interculturales sobre el patrimonio si las comunidades con cultura diferente están ya escindidas en otros temas que resuenan con lo externo. La consecuencia lógica es la confrontación entre unidad interna e influencia externa, que rompe políticamente la homogeneidad cultural remanente y promueve el fracaso de los esfuerzos de salvaguardia.

El caso de la educación intercultural presenta también problemas complejos como para pensar que no existen dilemas en su diseño e implementación. El primer tema surge no de lo político, sino de las características intrínsecas del proceso educativo moderno y en particular en comunidades indígenas tradicionales. Gonzalo Aguirre Beltrán, al escribir sobre el tema de la educación en contextos de contacto cultural, 
asevera que la educación encierra fuerzas contrarias -paradójicas- que enfrentan la conservación de lo tradicional, su renovación o su transformación (1992, p. 9). Es decir, se está hablando de que la reproducción de las herramientas cognitivas y tecnológicas que una comunidad valora para su desarrollo interno, se confrontan con su utilidad teórica y práctica en el medio externo. Desde luego que la apuesta de la educación intercultural es encontrar un mecanismo para lograr la convivencia de saberes con diferencia cultural o lingüística bajo principios de equidad y convivencia para el aprendizaje (CGEIB, 2017). Sin embargo, hay un balance complejo de resolver y que tiene que ver con lo que mencionó Aguirre Beltrán: el peso y foco en la reproducción de lo tradicional o de lo "nuevo" en función de las expectativas de la educación. Se parece un poco a los temas de traducción de una lengua a otra: se tiene que decidir en dónde estará el énfasis, si en la lengua de partida o en la lengua de llegada, pues una visión intermedia no es satisfactoria para los detalles finos de los extremos. En las políticas de educación intercultural en regiones indígenas el dilema es parecido, porque el énfasis en los contenidos internos se tensiona con la posibilidad de dispersar contenidos externos. Si bien se puede argumentar que los contenidos se convendrán en coordinación con las comunidades, como se mencionó a propósito de las obligaciones constitucionales, el dilema subsiste dado que la orientación externa empuja a la búsqueda de oportunidades “afuera”, mientras que la orientación hacia adentro empuja al fortalecimiento de la tradición interna. Como se ve, la tensión es la misma que en la traducción: empujar en una dirección reduce la dimensión opuesta; como precisara Levi-Strauss, la construcción de un escenario plenamente intermedio compromete la originalidad de los extremos.

El tema aquí es que en las referencias a las necesidades de las políticas educativas interculturales, la comunidad no siempre es homogénea. Existen diversas expectativas al interior que resuenan con las influencias de la vida exterior y que por lo tanto esperarían un balance mayor de lo externo frente a visiones más endógenas. El problema es que las políticas educativas del Estado tienen, como se reseñó, pulsiones uniformadoras de dimensión burocrática y presupuestal, Al mismo tiempo, al interior de la comunidad es muy complejo encontrar el balance deseado por cada uno de los actores a causa de la probable atomización. Así, el dilema entre necesidades individuales y pulsiones colectivas argumentado por Mouffe y Laclau tiene ecos paradójicos en el diseño y la implementación educativa. 
Un tema de la mayor importancia alrededor de las políticas interculturales se refiere a las características internas de la vida propia de las estructuras y los elementos culturales. Una cultura muta y se mantiene por "reglas" y procesos que duran decenas, cientos o miles de años, por lo que determinar la vigencia efectiva de un elemento resulta de la mayor complejidad. En este sentido, la implementación de una política que considere un elemento cultural como importante de valorar, puede estar lidiando con la obsolescencia de dicho elemento y, por ende, darle vida artificial a un proceso que ya no es relevante (Machuca, 2012). Es importante argumentar que la concurrencia de actores internos y externos en el desarrollo de las políticas de educación intercultural no garantiza que el acuerdo refleje la vigencia o la obsolescencia de una idea o proceso educativo. Como un ejemplo dramático, está el caso de que luego de casi 30 años de lucha de la etnia mazahua para tener una "universidad indígena", en su implementación desde el Gobierno del presidente Vicente Fox (2000-2006), se consideró que el apelativo de indígena en el nombre de la otorgada "Universidad Intercultural del Estado de México" no correspondía con la nueva realidad política del país. Así, los miembros de las comunidades indígenas tuvieron que aceptar la etiqueta, pues en la implementación estatal se consideró esa idea como ya obsoleta al margen de los intereses locales, privilegiando la denominación de "intercultural" por sobre la de "indígena" (Barquín, 2015, pp. 262-264). Al mismo tiempo existe la posibilidad contraria, es decir, que en términos técnicos un elemento cultural esté realmente desapareciendo porque ya no es funcional para los individuos de esa cultura. En ese sentido, elaborar políticas que lo consideren vigente porque desde el exterior o el interior se considera valioso, pueden resultar paradójicas ya que presionan el resultado hacia una región que es disfuncional en la cultura, lo que genera un resultado que es de naturaleza nostálgica o folclórica. Más aun, la implementación de tal política ocupa recursos y acciones públicas que pudieran ser utilizadas de mejor manera. En el caso de la educación intercultural que nos ocupa, un ejemplo de estas paradojas se ubica en el caso de la educación en lengua materna hacia comunidades hablantes. Si bien es de sobra conocido que la educación básica es más efectiva si se imparte en la lengua del hablante, es relevante considerar hasta qué nivel se debe seguir con ese proceso, en virtud de que en algunos casos la lengua está en un franco proceso de desaparición. Existen muchas lenguas en situaciones dramáticas en las que por el número total de hablantes, ya no son un medio de comunicación generalizado y por tanto la implementación de políticas educativas 
en la lengua no puede prolongarse a lo largo de todo el ciclo educativo porque resulta disfuncional y de gran dispendio presupuestal al margen de su obligación constitucional. Ejemplos de esto serían lenguas indígenas del norte de México con un número excepcionalmente bajo de hablantes: paipai (199 hablantes), cucapá (145 hablantes), kumiai (289 hablantes) y seri (764 hablantes), según datos del Instituto Nacional de Lenguas Indígenas (INALI, 2012). Un caso particular de lo anterior, son las ediciones de libros de texto para la educación primaria en lenguas indígenas, que publica y distribuye gratuitamente la Secretaría de Educación Pública del Gobierno Federal. En el caso del "mayo" hablado en Sonora y Sinaloa, se tiene contabilizada una comunidad total de 39616 hablantes de la lengua, de los cuales aproximadamente 4700 se ubican entre cinco y catorce años de edad y el resto, alrededor de 35 000, son mayores de edad (INALI, 2012). Como se ve, la proporción de adultos frente a niños y jóvenes es de casi 9 a 1 . Si no hubiera ningún cambio en el proceso de pérdida de la lengua, en una generación, el resultado sería de 4700 hablantes mayores y 500 menores. Esta situación obliga a cuestionarse hasta dónde se deben destinar recursos para este proceso y cuándo se debe parar. Resulta complicado emitir un juicio sin datos más precisos, pero es claro que tal política presenta ángulos paradójicos, porque no es factible regirse únicamente por la normatividad establecida, sino por lo mejor para la comunidad a mediano y largo plazo.

En el caso de la Universidad Intercultural del Estado de México, en virtud de la ausencia de profesionistas hablantes de las lenguas indígenas de la región, las materias regulares de cada licenciatura no se impartían de manera bilingüe; únicamente se daban clases de lenguas indígenas de la región para aquellos que no hablaran correctamente, solo las comprendieran o estuvieran iniciándose en su conocimiento (mazahua, otomí, tlahuíca y matlatzinca). El asunto es que para dos etnias en particular, tlahuícas y matlatzincas, el número de estudiantes es muy bajo, del orden del dos por ciento (Barquín, 2015). Pero la relación con el tema de la lengua es muy dramática, pues en el caso de la comunidad tlahuíca, se reportan 1548 hablantes y en el caso de la comunidad matlatzinca 1568 hablantes; en ambos casos ya no se reportan niños monolingües (INALI, 2018). El hecho de que las carreras que se imparten destinen tiempo específico para el estudio de las lenguas indígenas y su morfología, podría en el caso de estudiantes de estas etnias, enfocarse a otros campos de conocimiento más útiles para los individuos, en contraposición al ideal normativo. 
El tema de la relación entre educación en lengua y políticas interculturales tiene ángulos paradójicos como se ve, pues para lenguas indígenas en riesgo, la implementación de políticas de educación y preservación de la lengua colisiona con el dilema de preparar a los estudiantes para su inserción en algún nicho laboral. Es decir, resulta complejo determinar hasta dónde la educación básica debe impartirse en la lengua materna y hasta dónde parar. Por otro lado, las consideraciones sobre una cultura no terminan en la lengua y en ese sentido, es relevante la pertinencia de la educación intercultural que atiende a la generación de procesos y contenidos que están más allá de lo lingüístico y atañen a la dimensión cultural y el respeto de la identidad. El hilo conductor que va de la educación básica a la superior en comunidades indígenas, debe ser intercultural en atención a lo señalado anteriormente, pero en el caso de la educación bilingüe resulta fundamental determinar hasta dónde y en qué contenidos es útil a la comunidad para su reproducción al interior y su convivencia con el exterior.

Para culminar los ejemplos, se habían comentado los dilemas por la relación entre las expectativas externas y las internas en la implementación de las políticas y el difícil escenario para armonizar, en temas educativos, la preparación de los alumnos para su inserción laboral hacia afuera o hacia adentro. Un primer aspecto y que es relevante en la educación superior es el tema de la "fuga de cerebros". Toda política educativa universitaria de alcance nacional, en virtud de su expectativa de formar estudiantes que se acerquen a los límites del conocimiento, batalla con el conflicto de que los mejores productos de su esfuerzo pueden buscar su destino final en centros académicos o laborales externos al sistema. Lo anterior deriva en la fuga y la consecuente falla de la política nacional, pues se invierten recursos en individuos que no retribuirán a su comunidad de origen. Este fenómeno no es particular de la educación superior intercultural; el asunto es que la formación de agentes con alto nivel académico es en cualquier parte un elemento de tensión que resulta antagónico con la lógica de formar agentes para el desarrollo interno y no para su fuga.

En las comunidades indígenas y para el caso de la carrera de medicina tradicional o comunitaria que se imparte en algunas universidades interculturales de México, aparece un fenómeno que vale la pena analizar en virtud de sus ángulos paradójicos. Resulta interesante señalar el gran papel de la medicina tradicional en los contenidos 
de esta carrera, que se complementa con saberes de la medicina "científica”. Así se formaría un profesional que subsane los déficits de atención en salud, combinando ambas visiones: “... la salud comunitaria tiene en esencia, una formación con visión integral, científicamente capaz, humana en el trato y respeto a las costumbres y tradiciones de las comunidades indígenas" (Universidad Intercultural del Estado de Quintana Roo, 2018). Con ello, se forma un profesional que tomando en cuenta las diferencias culturales, puede dar atención en salud directamente en las comunidades indígenas y rurales (Universidad Intercultural del Estado de México, 2018). En este caso se estaría hablando de un efecto contrario al de la fuga, es decir, que la preparación de alto nivel de los egresados de las universidades interculturales los hace regresar y arraigarse en sus comunidades de origen o integrarse a estas aun cuando no sean originarios. La inserción en el nicho laboral sería exitosa para el egresado y benéfica para la comunidad. Sin embargo, hay un fenómeno paradójico en todo esto y que vale la pena analizar. Se entiende que en muchas de estas comunidades existe un médico tradicional. Algunos de ellos participaron o participan en colaboración con las universidades para la formación de los estudiantes, mediante la transmisión de los conocimientos que recibieron de sus mayores por vía oral o práctica. Estos mismos estudiantes van a regresar a las comunidades y competir laboralmente con los médicos tradicionales y se entiende que, por su componente científico y universitario, será en condiciones más favorables. Aquí surge una paradoja, porque siendo los estudiantes respetuosos de la medicina tradicional, van a competir ventajosamente con ella y con sus practicantes empíricos. Surgen varias preguntas: ¿A quién acudirá la población? ¿Desplazarán los doctores universitarios a los médicos tradicionales? ¿Surgirán tensiones entre estos? ¿Cuál será la relación entre el precio de los servicios: se elevará para los universitarios por la mejor preparación o bajará por la competencia con los médicos tradicionales? Desde luego que no son preguntas fáciles de responder, pero plantean paradojas en la implementación de las políticas educativas porque al impulsar el respeto y la comprensión de la cultura de las comunidades, introducen una gran tensión en su interior, pues a diferencia de otras carreras, estos egresados sí compiten directamente con miembros de la comunidad. No solo eso, sino que a mediano o largo plazo, puede ocurrir un fenómeno contrario al espíritu constitucional de defensa y fortalecimiento de las culturas tradicionales que fundamentó la política: que por la mejor preparación de los egresados de las universidades interculturales, se vaya debilitando la práctica de la medicina tradicional y se rompa la cadena de 
transmisión intergeneracional, ya que estudiar en la universidad es "mejor" y más "rentable" que con el curandero del lugar, que carece de la certificación universitaria. La ocurrencia de esta ruptura producirá la paradoja de que para aprender medicina tradicional, habrá que ir a la universidad porque en la comunidad de origen "ya no existe”. Desde luego que este escenario es profundamente dramático y el más extremo, sin embargo, hay que señalar una de las advertencias sobre los efectos no intencionales que se mencionaron arriba y que tiene que ver con la "gran intervención". Si tal consecuencia es factible de ocurrir, cuando aparezcan sus manifestaciones podría ser muy tarde para corregir, pues la pérdida del interés en la práctica y transmisión de la medicina tradicional se habrá ocasionado por la presencia de agentes internos con preparación externa. Es decir, contrario al efecto de la "fuga de cerebros", aquí lo que ocurre es que el deseo de que los profesionales se queden en sus comunidades es exitoso, pero dañino a la cultura tradicional que es justo lo que animó la política de educación intercultural. Los efectos de corto plazo y las dinámicas normativas que impulsan los diseńos concretos, generan en el mediano plazo efectos no intencionales y paradojas normativas sobre los resultados deseables más profundos.

\section{ConClusión}

En esta reflexión se presentó una exposición panorámica de varios temas que inciden en el desarrollo de las políticas interculturales y que merecen ser analizados con reposo, pues como se definió al inicio, en muchos casos se esconde el riesgo de resultados paradójicos. Se mencionó cómo es que la política misma encierra dilemas insolubles y por lo tanto, la inestabilidad en sus dinámicas y resultados arroja soluciones provisionales a causa de los antagonismos persistentes. Esta característica no es distinta en las soluciones que un Estado busca aplicar cuando implementa políticas en comunidades con diferencias culturales, pues dicha circunstancia es un antagonismo más en términos políticos. No obstante, dada su naturaleza de estructura mayor, la diferencia cultural debe ser manejada con gran serenidad porque atañe a la inteligibilidad entre ambas partes. Por ello, la idea de interculturalidad en las políticas públicas es un insumo sano y necesario. Sin embargo, esta inclusión no supone el fin de los conflictos ni tampoco, y esto es fundamental, el fin de los efectos no intencionados o perversos que aparecen en cualquier política y que en 
la teoría sobre el tema están muy bien descritos. Ese fue el espíritu al ilustrar con algunos casos la implementación de políticas interculturales. Los argumentos de lo que resulta paradójico en los efectos ocurridos o por ocurrir, no son sentencias de lo mal planeado o mal instrumentado, sino llamadas de atención por el bajo control que los humanos tienen sobre la manipulación de la realidad. En muchas ocasiones, estas dificultades de origen se contraponen a la gran confianza y esperanza generadas por las soluciones imaginadas que, ancladas en la justificación axiológica y la coyuntura política, desdeñan la terca persistencia de los efectos no intencionales en la implementación de políticas, en este caso, de dimensión intercultural.

\section{REFERENCIAS}

Aguilar Villanueva, L. (2000). La hechura de las políticas (3a ed.). México D. F., México: Miguel Ángel Porrúa.

Aguirre Beltrán, G. (1992). Teoría y práctica de la educación indígena. Obra polémica, $X$. México: Universidad Veracruzana-INI-FCE.

Arendt, H. (1997). ¿Qué es la politica? Barcelona, España: Paidós.

Ávila Domínguez, R. (2018). Interculturality in Health: The pending issue of the Mexican Health System. Mexican Journal of Medical Research, 6(12), 6-9. DOI: https://doi.org/10.29057/mjmr.v6i12.3163

Barquín Cendejas, A. (2015). Antropología y poder politico. El ejercicio de poder en las políticas de educación intercultural. México D. F., México: INAH.

Bartolomé, M. A. (2006). Procesos interculturales: antropología politica del pluralismo en América Latina. México D. F., México: Siglo Veintiuno Editores. 
Bertely, M. (coord.). (2009). Sembrando nuestra educación intercultural como derecho: la nueva escuela intercultural en Chiapas. México D. F., México: CIESASEdiciones Alcatraz.

Bolaños, M., y Barquín, A. (2014). Entrevista con Rubisel Nigenda, patrón de los parachicos. Diario de Campo, (2). Tercera época, abril-junio.

Bonfil Batalla, G. (1991). Pensar nuestra cultura. México D. F., México: Alianza Editorial.

Centro de Estudios Sociales y de Opinión Pública - CESOP. (2015). Políticas públicas interculturales para um mundo diverso. México D. F., México: CESOPCámara de Diputados.

Collins, R. (1996). Cuatro tradiciones sociológicas. México D. F., México: UAM.

Constitución Política de los Estados Unidos Mexicanos. (1917). Recuperado de http://www.ordenjuridico.gob.mx/Constitucion/cn16.pdf

Coordinación General de Educación Intercultural Bilingüe - CGEIB. (2006). Universidad intercultural. Modelo educativo. México D. F., México: SEP-CGEIB.

Coordinación General de Educación Intercultural Bilingüe - CGEIB. (2017). ABC de la interculturalidad. Qué es el enfoque intercultural en la educación. México D. F., México: CGEIB. Recuperado de https://www.gob.mx/sep/articulos/ sabes-en-que-consiste-la-educacion-intercultural

Cruz Rodríguez, E. (2014). ¿Qué deben ser las políticas interculturales? Ciencia e Interculturalidad, 15(2), 68-80.

Cruz Rodríguez, E. (2015). La interculturalidad en las políticas de educación intercultural. Praxis \& saber, 6(12), 191-207. 
Diario de Campo. (2014). Los dilemas de la salvaguardia. A diez años de la Convención para la salvaguardia del patrimonio cultural inmaterial. Diario de Campo, (2), abril-junio.

Díaz Polanco, H. (2006). Elogio de la diversidad. Globalización, multiculturalismo y etnofagia. México D. F., México: Siglo Veintiuno Editores.

Dietz, G. (2003). Multiculturalismo, interculturalidad y educación. Una aproximación antropológica. Granada, España: CIESAS-Universidad de Granada.

Dror, Y. (2000). Salir del paso, ¿'ciencia’ o inercia? En L. Aguilar Villanueva, La hechura de las politicas (3a ed.) (pp. 255-264). México D. F., México: Miguel Ángel Porrúa.

Etzioni, A. (2000). La exploración combinada: un tercer enfoque en la toma de decisiones. En L. Aguilar Villanueva, La hechura de las políticas (3a ed.) (pp. 265282). México D. F., México: Miguel Ángel Porrúa.

Fleckman, J. M., Dal Corso, M., Ramírez, S., Begalieva, M., y Johnson, C. C. (2015). Intercultural competency in public health: a call for action to incorporate training into public health education. Frontiers in Public Health, 3, 1-7.

Flores Mercado, G. (2014). "Y con la pirekua ni siquiera nos preguntaron...”. La declaración de la pirekua como patrimonio cultural inmaterial de la humanidad. Diario de Campo, (2). Tercera época, abril-junio.

Huerta Morales, G. (2016). Procesos interculturales y construcciones sociales: apuntes para una antropología de las políticas públicas. Iberoamérica Social: revista-red de estudios sociales, VI, 118-134.

Instituto Nacional de Lenguas Indígenas - INALI. (2012). Lenguas indigenas nacionales en riesgo de desaparición. México. Recuperado de https://site.inali. 
gob.mx/publicaciones/libro_lenguas_indigenas_nacionales_en_riesgo_de_ desaparicion/libro_lenguas_indigenas_nacionales_en_riesgo_de_desaparicion. html

Instituto Nacional de Lenguas Indígenas - INALI. (2018). Micrositio. https://site. inali.gob.mx/Micrositios/estadistica_basica/estadisticas2015/index_indicadores_ basicos_agrupacion_linguistica_2015.html

Laclau, E., y Mouffe, C. (2004). Hegemonía y estrategia socialista. Hacia una radicalización de la democracia (2a ed.). Buenos Aires, Argentina: FCE.

Lévi-Strauss, C. (2000). Raza y cultura. Introducción de Manuel Garrido. Madrid, España: Cátedra.

Lindblom, C. ([1959] 2000). La ciencia de 'salir del paso'. En L. Aguilar Villanueva, La hechura de las politicas (3a ed.) (pp. 201-225). México D. F., México: Miguel Ángel Porrúa.

Machuca, A. (2012). Notas sobre la música tradicional como patrimonio cultural inmaterial. Documento de trabajo presentado al Seminario sobre Salvaguardia del Patrimonio Musical. DEAS-INAH, México D. F., México.

Matto, D. (coord.). (2008). Diversidad cultural e interculturalidad en educación superior. Experiencias en América Latina. Caracas, Venezuela: IESALC-Unesco.

Mendoza Zuany, R. (2013). Educación intercultural y la gestión de la diversidad en regiones indígenas de México: el caso de la educación superior. Entreciencias. 1(2), 237-247.

Merino, M. (2013). Politicas públicas. Ensayo sobre la intervención del Estado en la solución de problemas públicos. México D. F., México: CIDE. 
Merton, R. (1936). The unanticipated consequences of purposive social action. American Sociological Review, 1(6), 894-904

Ministerio de Cultura. (2009). Memoria de los seminarios de gestión local intercultural. Quito, Ecuador: Ministerio de Cultura.

Monroy Gaytán, F. (2017). La evaluación de los indicadores de interculturalidad en los programas de salud dirigidos a la población indígena y la importancia de la comunicación intercultural. Revista de Programas y Politicas Públicas, (8), 71-89. DOI: $10.5944 /$ reppp.8.2017.15952

Mouffe, C. (2012). La paradoja democrática. El peligro del consenso en la politica contemporánea. Barcelona, España: Gedisa.

Orozco, J. L. (1978). La pequeña ciencia. Una critica de la ciencia política norteamericana. México D. F., México: Fondo de Cultura Económica.

Parsons, W. (2007). Políticas públicas. Una introducción a la teoría y la práctica del análisis de politicas públicas. México D. F., México: Flacso-Miño y Dávila Editores.

Pérez Ruiz, M. (2014). El Día de Muertos como patrimonio. Los dilemas de una convención en Michoacán. Diario de Campo, (2). Tercera época, abril-junio.

Rebollo, M. (2017). Un acercamiento al patrimonio cultural inmaterial, su salvaguardia y patrimonialización. Campos, 5(1-2), 175-209. DOI: http://dx.doi. org/10.15332/s2339-3688.2017.0001.07

Rodríguez Cruz, M. (2015). ¿Interculturalidad para todos? Políticas públicas y prácticas educativas en Ecuador. Periferia, 1(20), 110-148. 
Sartori, G. (2002). La política. Lógica y método en las ciencias sociales. (2a ed.). México D. F., México: Fondo de Cultura Económica.

Schmitt, C. (1998). El concepto de lo político. Madrid, España: Alianza Editorial.

Unesco. (2004). Convención para la salvaguardia del patrimonio cultural inmaterial. Diario de Campo (separata), agosto de 2004.

Universidad Intercultural del Estado de Quintana Roo. (2018). Plan de estudios de la Licenciatura en Salud Comunitaria. Recuperado de http://www.uimqroo.edu. $\mathrm{mx} /$ programa-SC.php

Universidad Intercultural del Estado de México. (2018). Plan de estudios de la Licenciatura en Salud Intercultural. Recuperado de http://uiem.edu. $\mathrm{mx} /$ ?page_id $=1687$

Weber, M. (1998). El político y el cientifico. Madrid, España: Alianza Editorial.

Zapata-Barrero, R. (2016). Exploring the foundations of the intercultural policy paradigm: a comprehensive approach. Identities, 23(2), 153-173. https://doi.org /10.1080/1070289X.2015.1006523 